Supporting Information

\title{
Intrinsically photo-polymerizable dynamic polymers derived from a natural small molecule
}

Chen-Yu Shi, ${ }^{1}$ Qi Zhang, ${ }^{1}$ Bang-Sen Wang, ${ }^{1}$ Meng Chen, ${ }^{1}$ Da-Hui Qu ${ }^{1 *}$

${ }^{1}$ Key Laboratory for Advanced Materials and Joint International Research Laboratory of Precision Chemistry and Molecular Engineering, Feringa Nobel Prize Scientist Joint Research Center, Frontiers Science Center for Materiobiology and Dynamic Chemistry, School of Chemistry and Molecular Engineering, East China University of Science and Technology, 130 Meilong Road, Shanghai 200237, China.

Email: Da-Hui Qu: dahui_qu@ecust.edu.cn 


\section{Content:}

\section{Materials and methods}

\section{Supporting figures}

Figure S1. XRD spectra of TA monomer, thermal poly(TA), and photo-poly(TA).

Figure S2. (a) Polarized optical microscopy images and (b) photographs of TA monomer, thermal poly(TA) and photo-poly(TA).

Figure S3. UV-Vis absorption spectra of the photopolymerization process of TA monomers at $365,380,400 \mathrm{~nm}$.

Figure S4. ATR-IR spectra of TA monomer, thermal poly(TA) and photo-poly(TA).

Figure S5. (a) ${ }^{1} \mathrm{H}$ NMR spectra (400 MHz, 298K, $\mathrm{CDCl}_{3}$ ), (b) ATR-IR spectra, and (c)Raman spectra of TMe monomer, and photo-poly(TMe); (d) Gel Permeation Chromatography (GPC) of photo-poly(TMe). (Solvent: THF)

Figure S6. Photographs of solubility of (a) Photo-irradiated poly(TA) and (b) poly(TADIB-Fe) copolymer in different solvents.

Figure S7. Schematic representation of the synthesis route and structure of the photopoly(TA-DIB-Fe) copolymer.

Figure S8. Frequency-dependent rheology curves and photographs of thermal and photo-copolymers with different $\mathrm{TA} / \mathrm{Fe}$ molar ratios.

Figure S9. XPS of thermal and photo-copolymers.

Figure S10. Frequency-dependent rheology curves of photo-copolymers with different (a) TA/Zn and (b) TA/Cu molar ratios.

Figure S11. The photograph of thermal copolymer adhesion performance on different substrates.

Figure S12. Adhesion strength comparison diagram of this work and previous works on supramolecular adhesives.

Figure S13. Time-dependent shear strength curve of photo-copolymer underwater. The shear strength stabilized at $3 \mathrm{MPa}$ after 5 days.

Figure S14. ${ }^{1} \mathrm{H}$ NMR spectra of five cycles of photo polymerization-thermal depolymerization. (400 MHz, 298K, [D6]acetone)

Figure S15. Optical microscopy images of damaged and healed coatings at $20^{\circ} \mathrm{C}$.

Figure S16. UV-Vis transmission spectrum of photo-curing poly(TA) coating. 


\section{Materials and methods}

General Information: All the reagents were purchased from Adamas ${ }^{\circledR}$ beta, TCI and Aldrich. The key $( \pm$ )- $\alpha$-thioctic acid (TA) (Reagent Grade, 99\%) was used as received from Adamas ${ }^{\circledR}$ beta. Chemicals were weighed on analytical balances (METTLERTOLEDO, ME204T/02). High power LED lamp source (Beijing Perfectlight: PLSLED100C) of different wavelengths $(365 \mathrm{~nm}-450 \mathrm{~nm})$ with $50 \mathrm{~W}$ power was utilized to obtain photo-polymerized polymers. Nuclear magnetic resonance (NMR) spectrum was tested by Brüker AV-400 spectrometer using tetramethylsilane to be the internal standard. X-ray diffraction (XRD) patterns were obtained on a rotating anode X-ray powder diffractometer $(18 \mathrm{KW} / \mathrm{D} / \mathrm{max} 2550 \mathrm{VB} / \mathrm{PC})$ equipped with a copper target $18 \mathrm{KW}(450 \mathrm{~mA})$, a fully automated curved (plate) crystal graphite monochoromator and a programmed variable slit system. The Raman spectra were recorded by using a Laser Micro-Raman Spectrometer (Renishaw, I0.2/cm/invia reflex) equipped with a high performance grade Leica DMLM microscope and a $514 \mathrm{~nm}$ excitation wavelength. Fourier transform infrared spectrometer (FT-IR) (Thermo Nicolet Corporation; 7800$350 / \mathrm{cm} 0.01 / \mathrm{cm} / 6700$ ) was used to analyze the copolymer samples. Gel Permeation Chromatography (GPC) (Waters Corporation; Waters1515) and Matrix-Assisted Laser Desorption/ Ionization Time of Flight Mass Spectrometry (MALDI-TOF-MS) (Singapore ABS Corporation; $400 \sim 500000 \mathrm{Da} / 4800$ plus) were used to determine the polymer molecular weight distribution. The valence state of iron was determined with X-ray photoelectron spectroscopy (XPS) (Thermo Fisher Corporation; resolution ratio > $3 \mu \mathrm{m} / \mathrm{ESCALAB} 250 \mathrm{Xi})$. The mechanical properties of the polymer films were measured by an HY-0580 tension machine (HENGYI), and the thermal stability was measured by thermogravimetric analysis (Mettler Toledo TGA/SDTA851, heating rate $\left.=5^{\circ} \mathrm{C} \mathrm{min}^{-1}\right)$. The thermal properties were measured by differential scanning calorimetry (TA instruments; modulated DSC2910, 1090B, ramp rate $=5^{\circ} \mathrm{C} \mathrm{min}^{-1}$ ). The rheological experiments were performed by rotational rheometer (TA InstrumentsWaters LLC; DHR-2). The polarized optical microscopy images were obtained on a typical optical microscopy equipped with two polarizers. 


\section{Supporting figures}

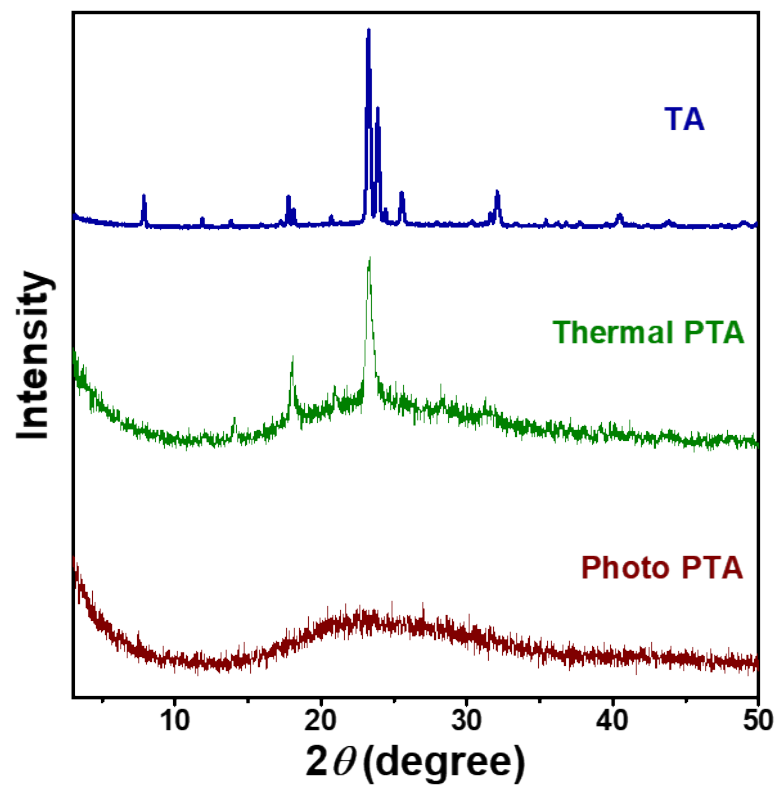

Figure S1. XRD spectra of TA monomer, thermal poly(TA), and photo-poly(TA).

\section{TA monomer Thermo-Poly(TA) Photo-Poly(TA)}

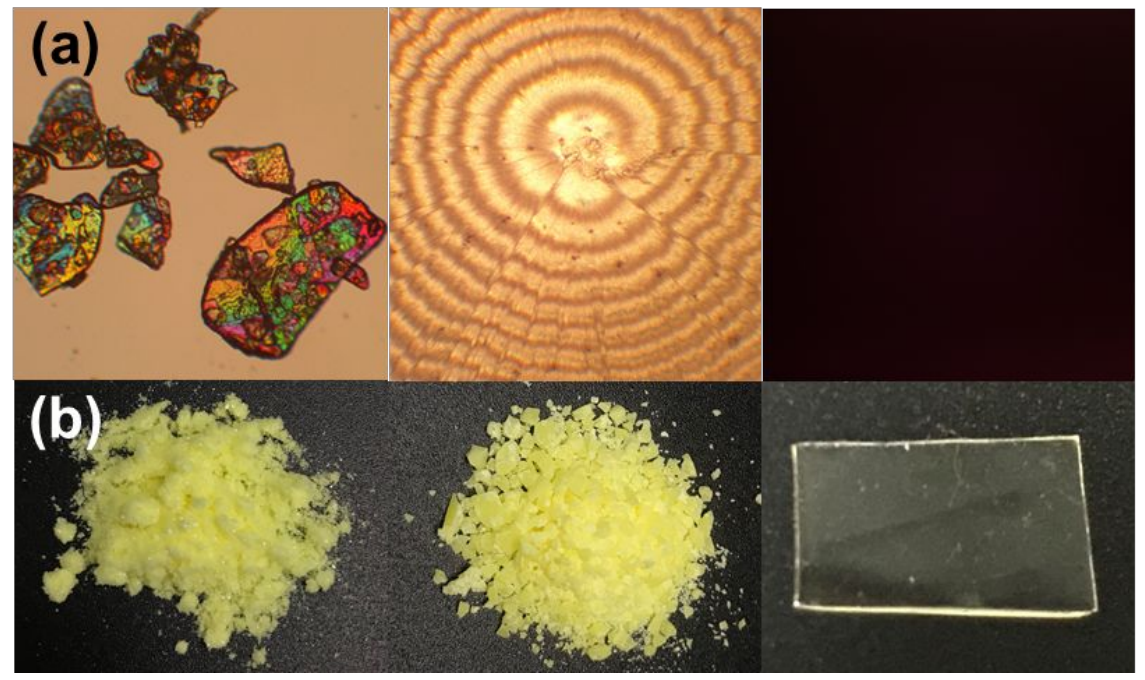

Figure S2. (a) Polarized optical microscopy images and (b) photographs of TA monomer, thermal poly(TA) and photo-poly(TA). 

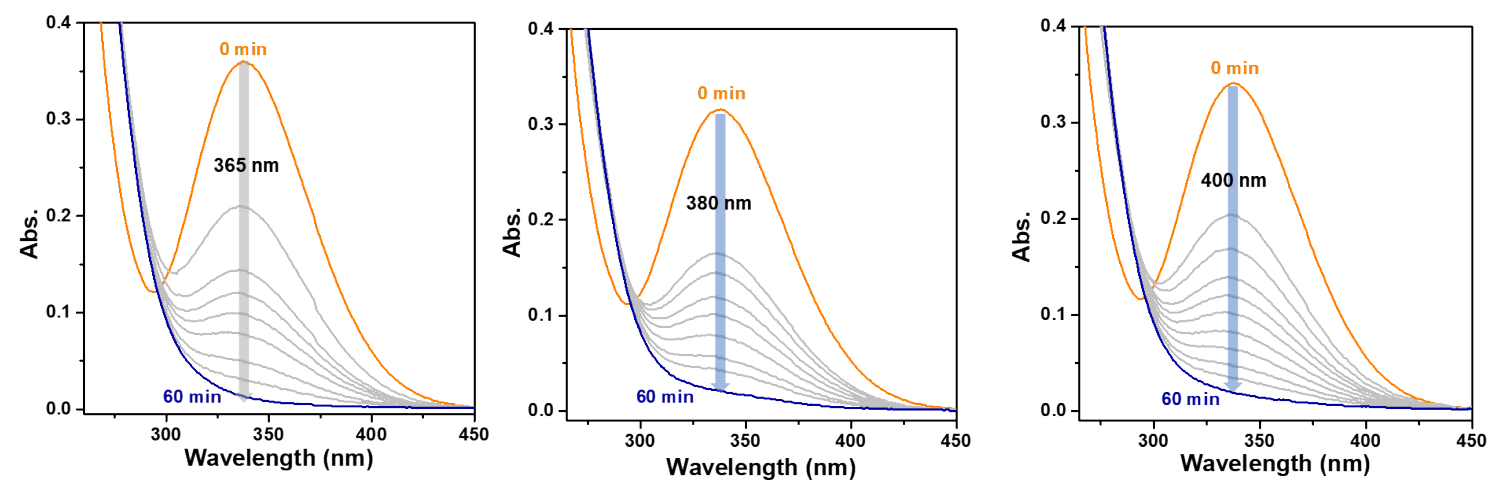

Figure S3. UV-Vis absorption spectra of the photo-polymerization process of TA monomers at $365,380,400 \mathrm{~nm}$.

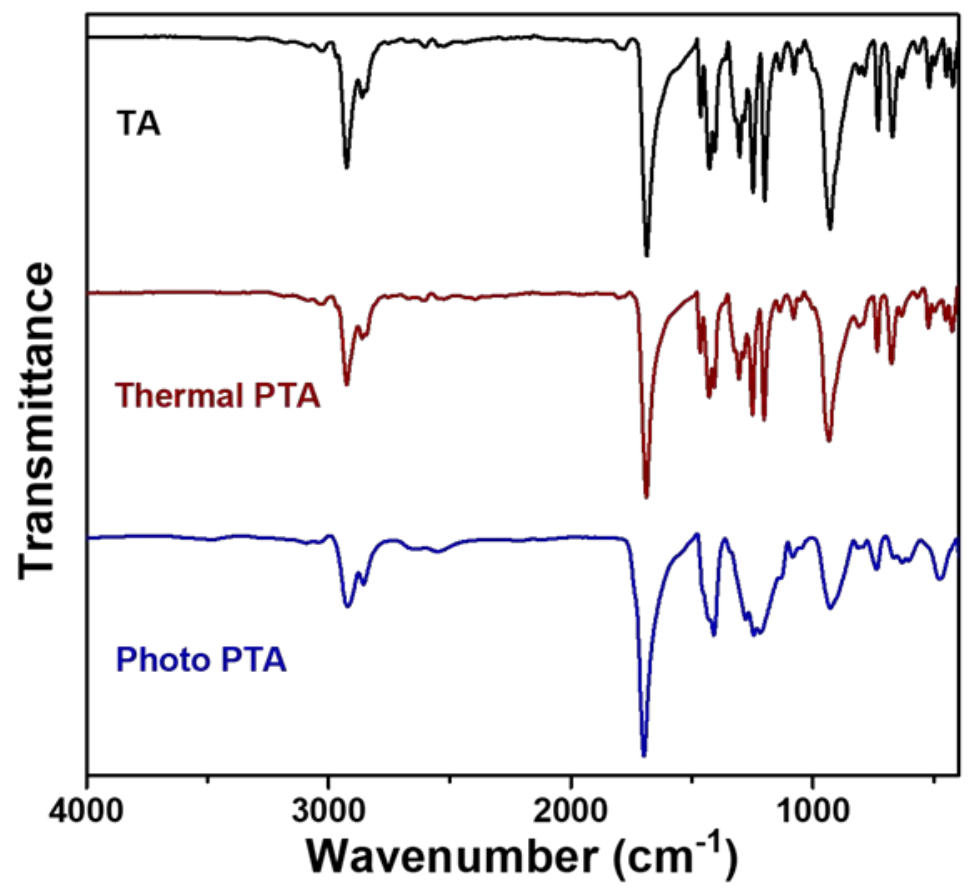

Figure S4. ATR-IR spectra of TA monomer, thermal poly(TA) and photo-poly(TA). 
(a)

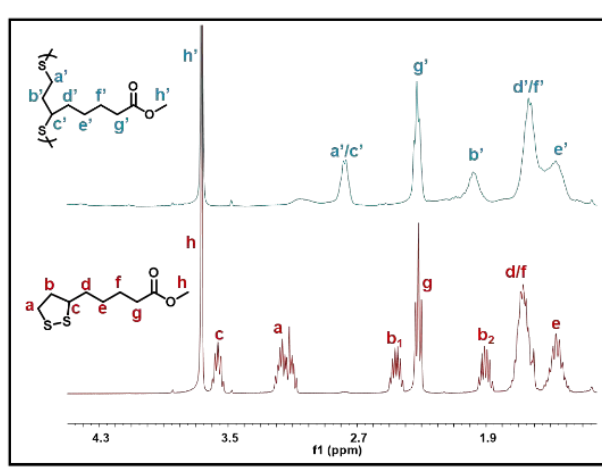

(c)

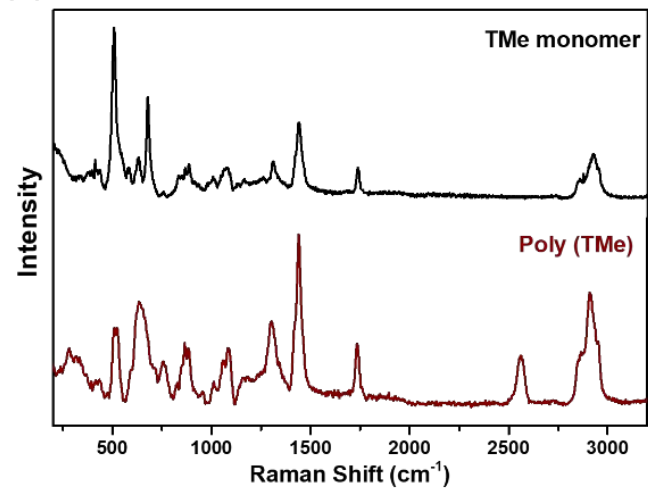

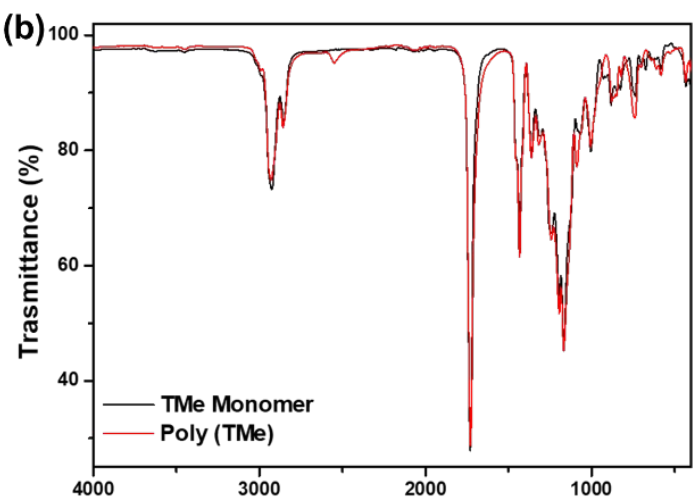

(d)

Wavenumber $\left(\mathrm{cm}^{-1}\right)$

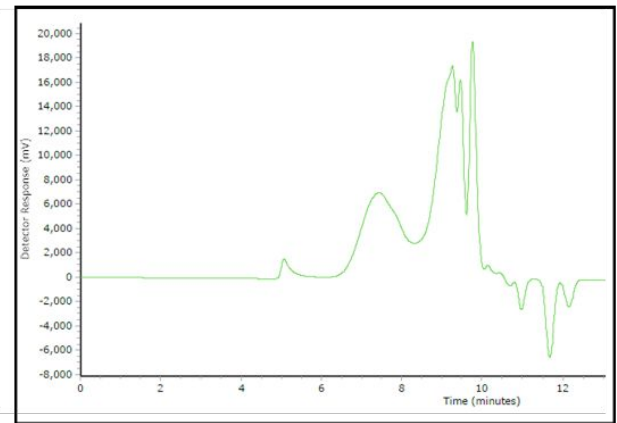

Figure S5. (a) ${ }^{1} \mathrm{H}$ NMR spectra $\left(400 \mathrm{MHz}, 298 \mathrm{~K}, \mathrm{CDCl}_{3}\right)$, (b) ATR-IR spectra, and (c)Raman spectra of TMe monomer, and photo-poly(TMe); (d) Gel Permeation Chromatography (GPC) of photo-poly(TMe). (Solvent: THF)

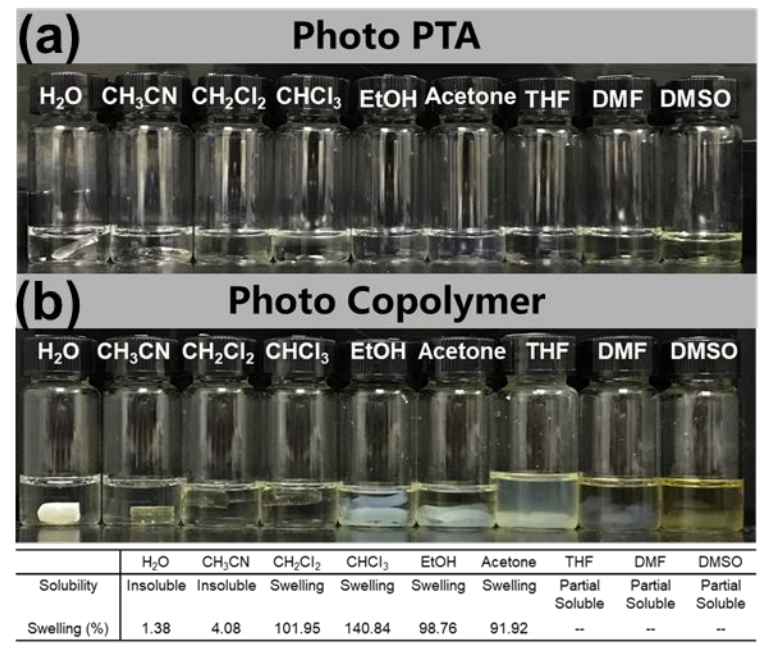

Figure S6. Photographs of solubility of (a) Photo-poly(TA) and (b) poly(TA-DIB-Fe) copolymer in different solvents. (Concentration: $50 \mathrm{mg} / \mathrm{ml}$; Temperature: $25^{\circ} \mathrm{C}$ ) 


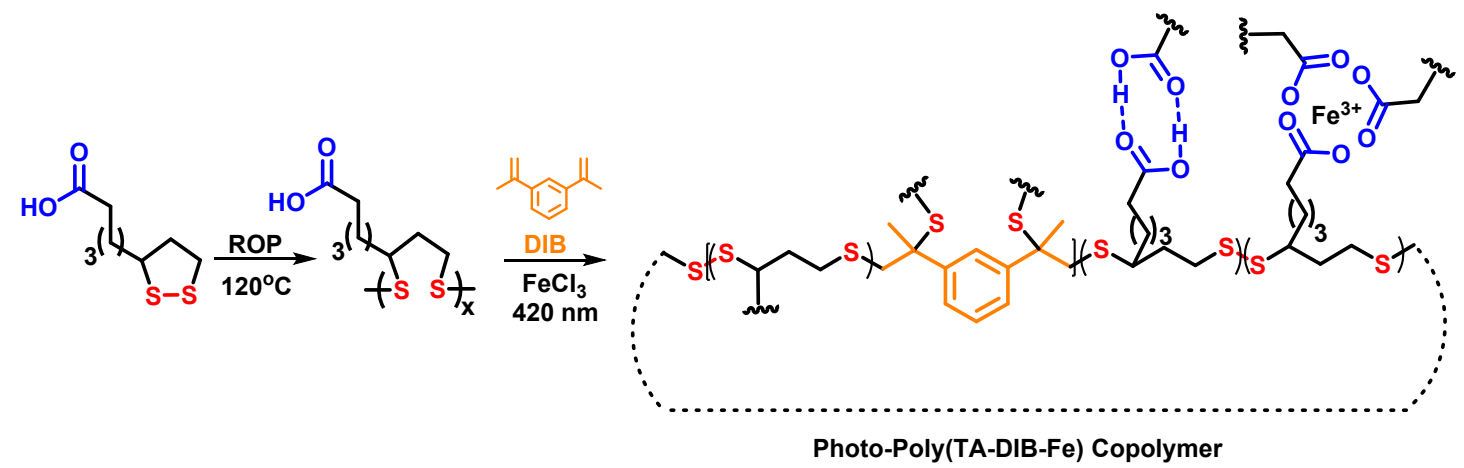

Figure S7. Schematic representation of the synthesis route and structure of the photopoly(TA-DIB-Fe) copolymer.
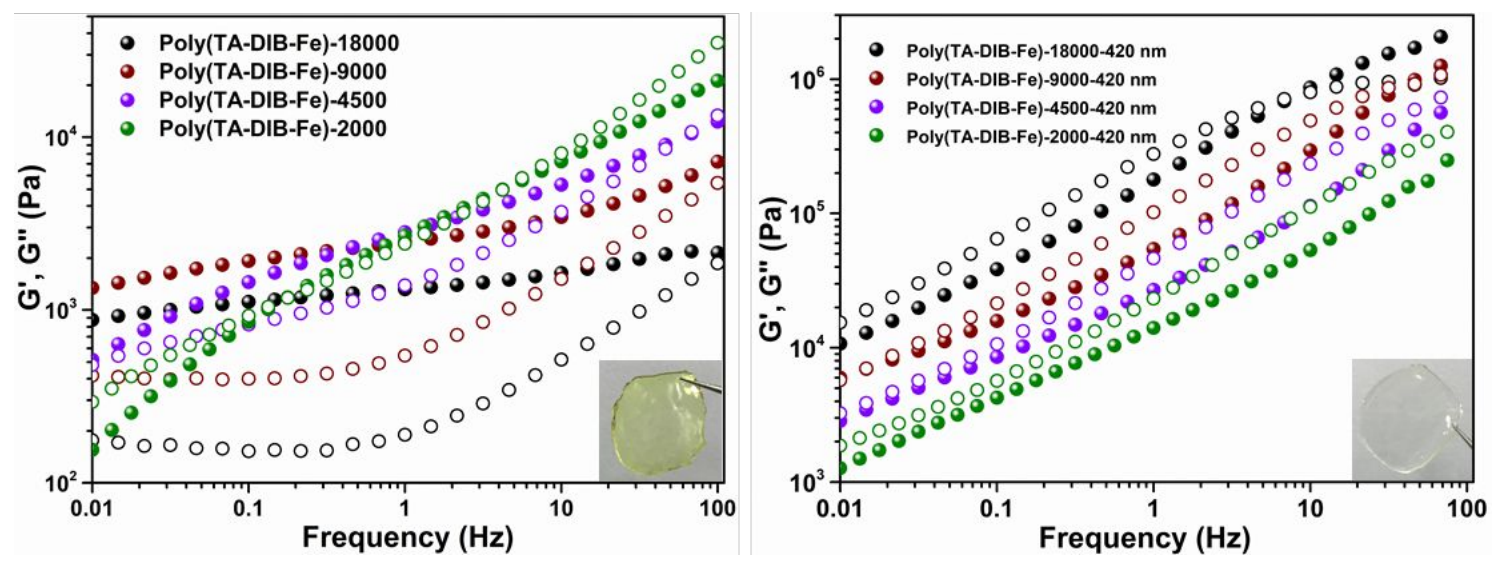

Figure S8. Frequency-dependent rheology curves and photographs of thermal and photo-copolymers with different $\mathrm{TA} / \mathrm{Fe}$ molar ratios. 

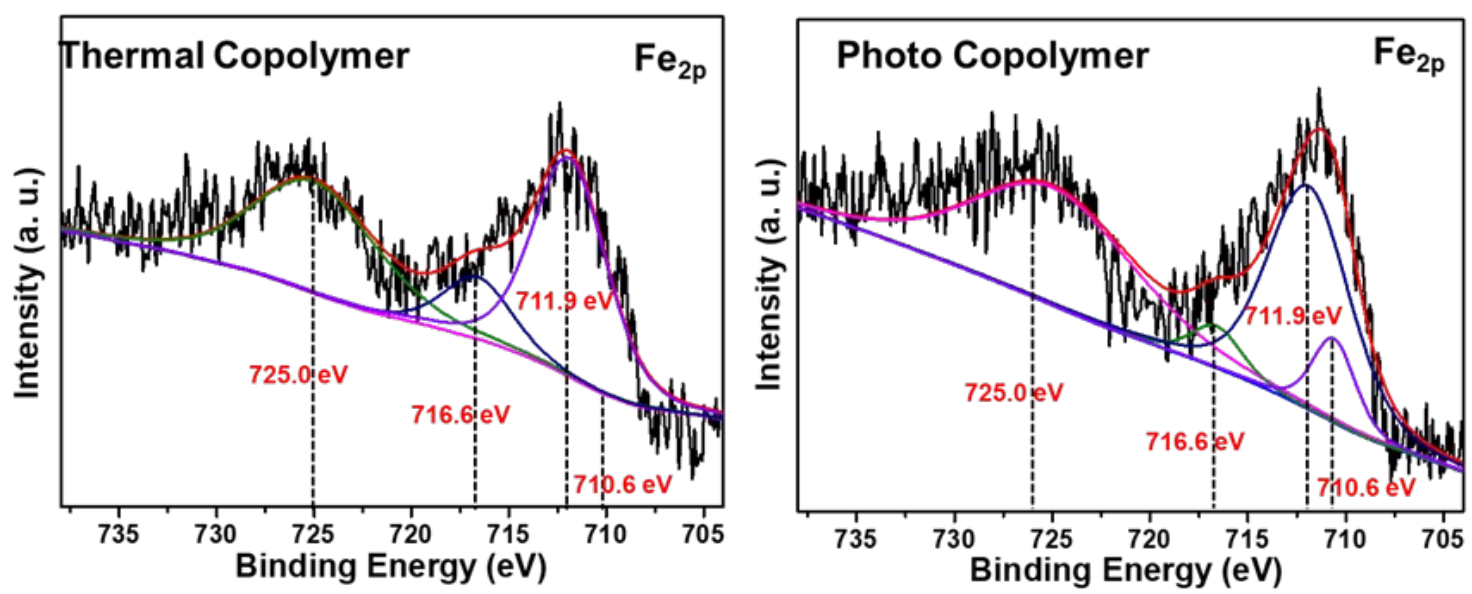

Figure S9. XPS of thermal and photo-copolymers. The peaks at $725.0 \mathrm{ev}$ and 711.9 ev were ascribed to the spin-splitting of Fe(III)2p, and the peaks at $716.6 \mathrm{ev}$ and 710.6 ev were ascribed to the spin-splitting of Fe(II)2p.
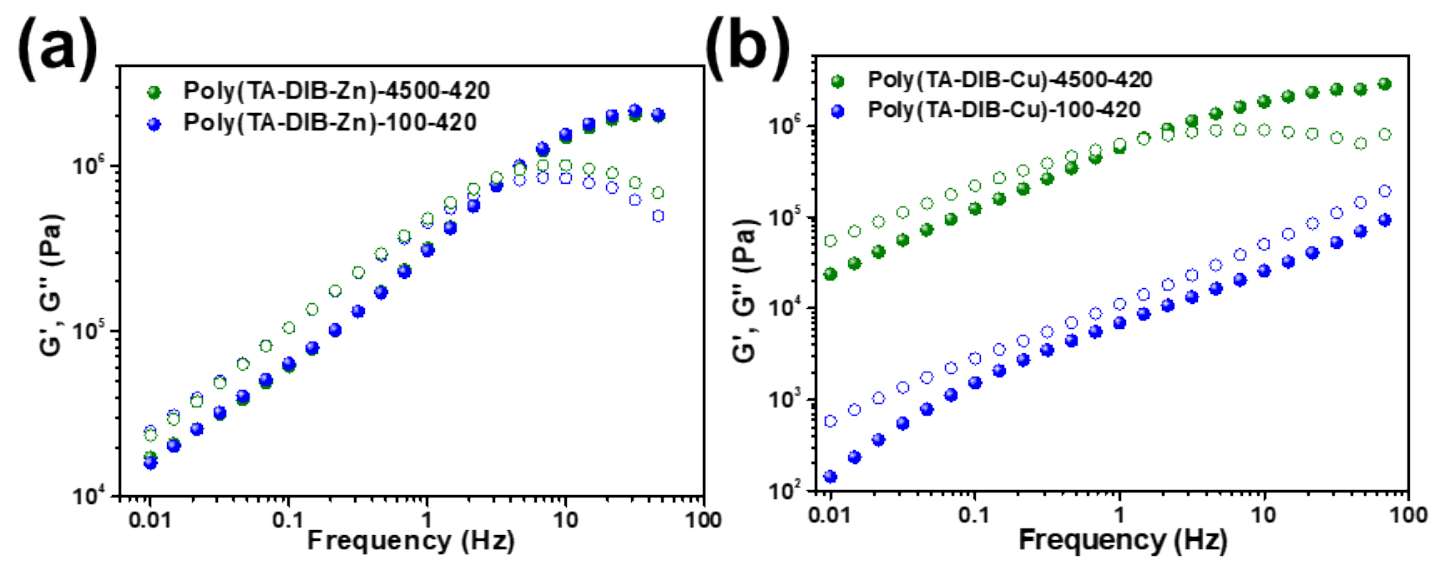

Figure S10. Frequency-dependent rheology curves of photo-copolymers with different (a) TA/Zn and (b) TA/Cu molar ratios. The mechanical modulus of poly(TA-DIB-Zn) with different ratios of photo-stable $\mathrm{Zn}(\mathrm{II})$ maintained consistent, while the mechanical modulus of poly(TA-DIB-Cu) with different ratios of photoreduced $\mathrm{Cu}(\mathrm{II})$ decreased with the increase of $\mathrm{Cu}(\mathrm{II})$ content. 


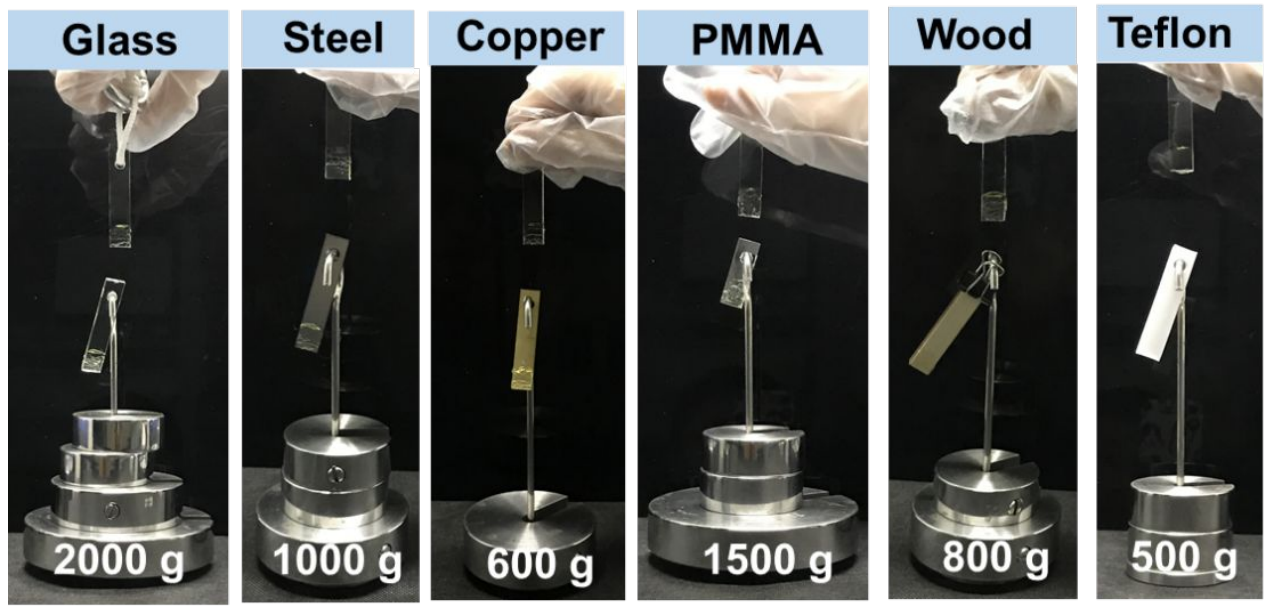

Figure S11. The photograph of thermal copolymer adhesion performance on different substrates.

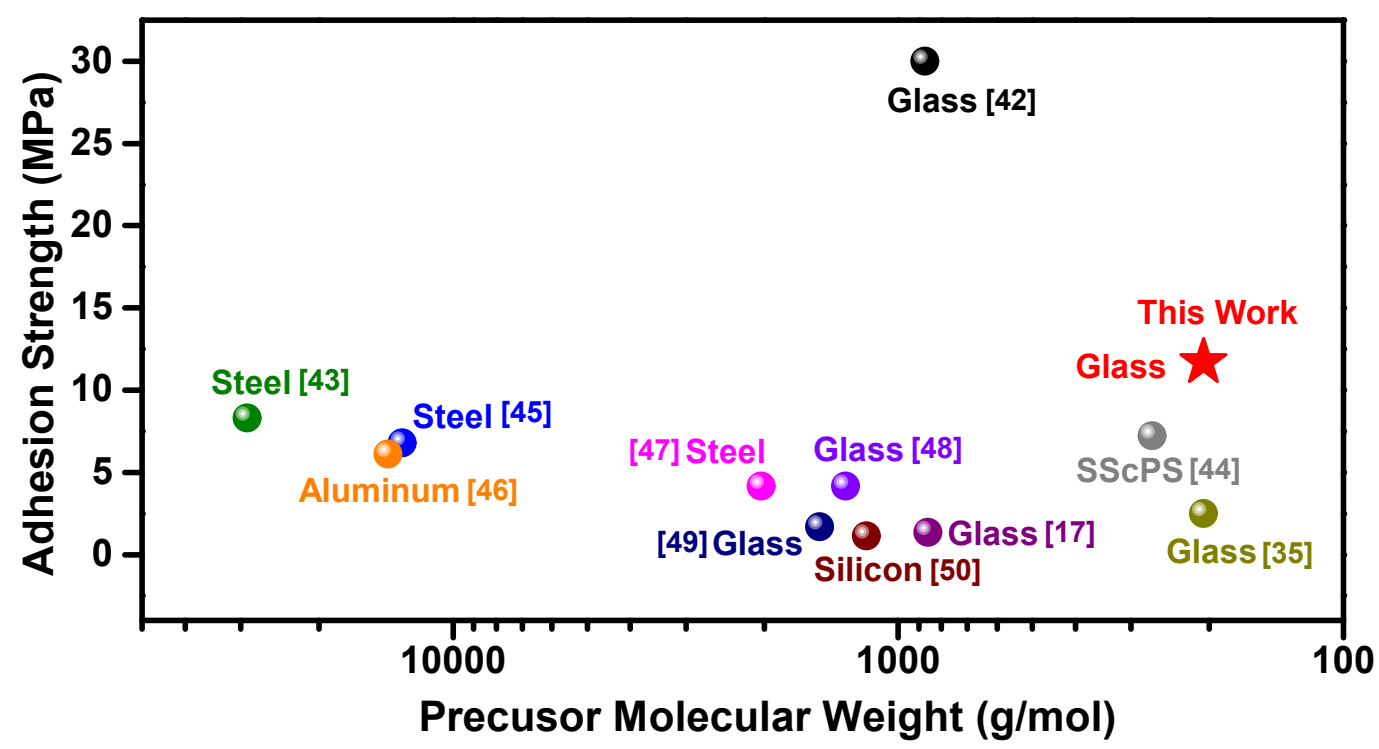

Figure S12. Adhesion strength comparison diagram of this work and previous works on supramolecular adhesives 


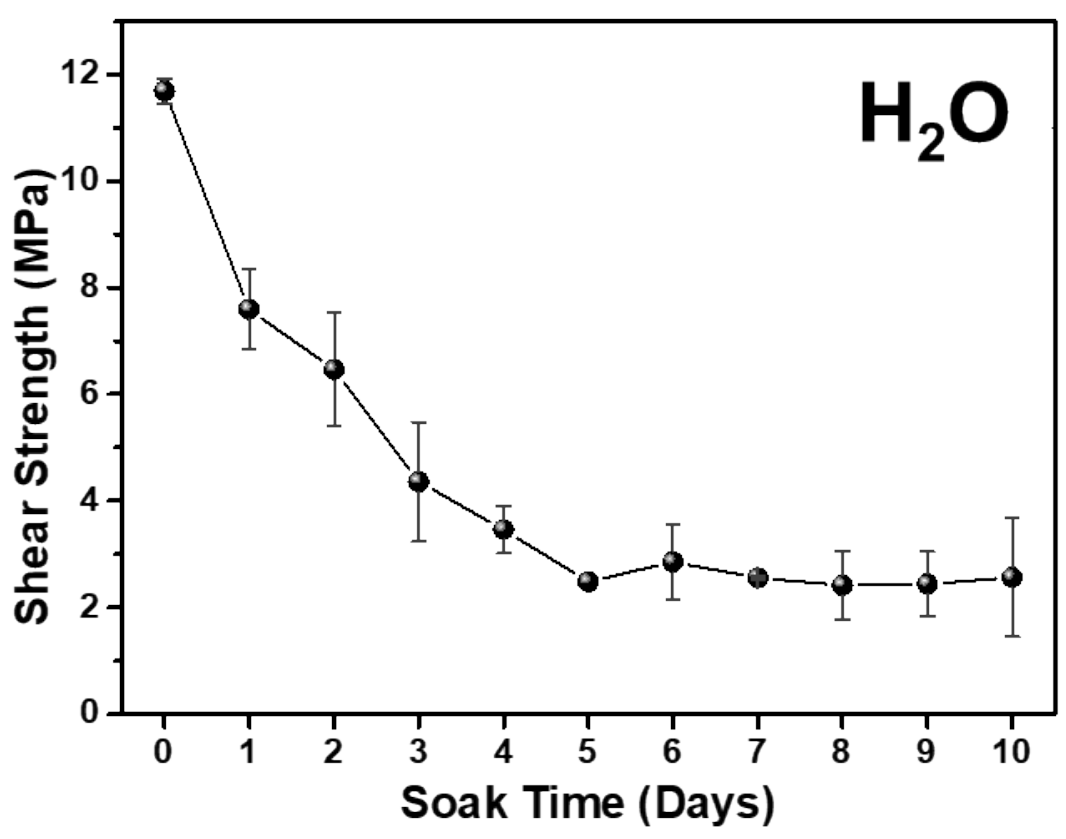

Figure S13. Time-dependent shear strength curve of photo-copolymer underwater. The shear strength stabilized at $3 \mathrm{MPa}$ after 5 days.

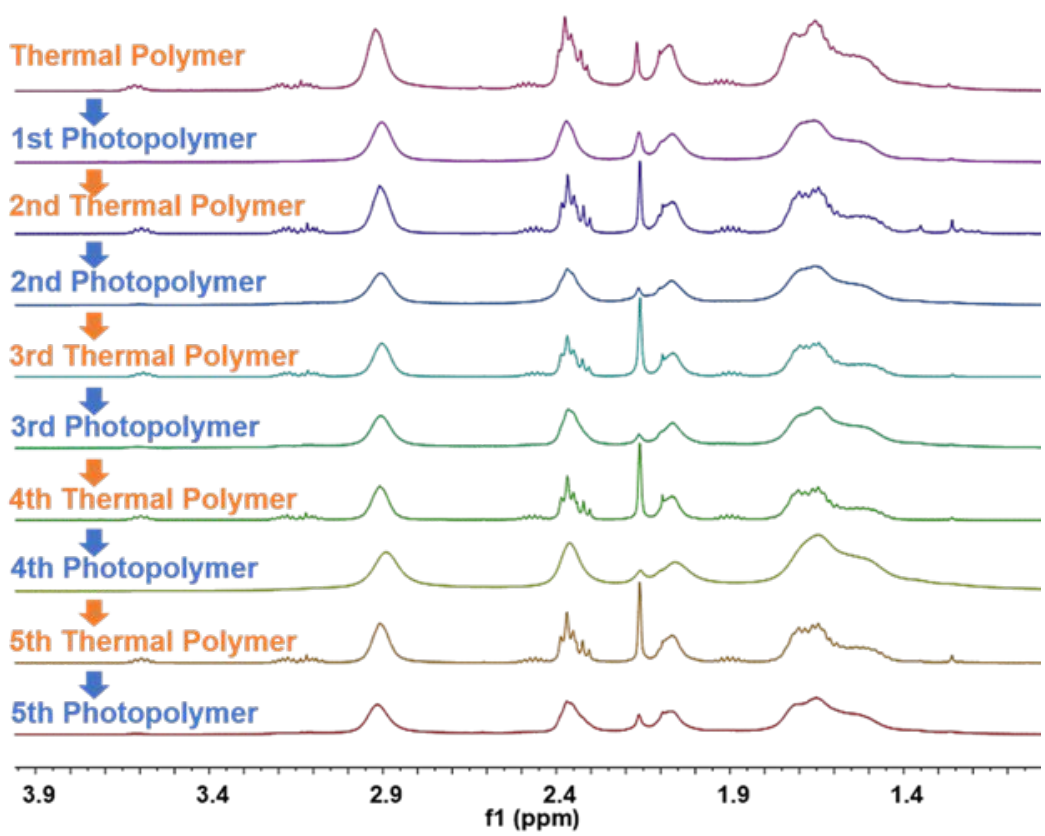

Figure S14. ${ }^{1} \mathrm{H}$ NMR spectra of five cycles of photo polymerization-thermal depolymerization. (400 MHz, $298 \mathrm{~K}$, [D6]acetone) 


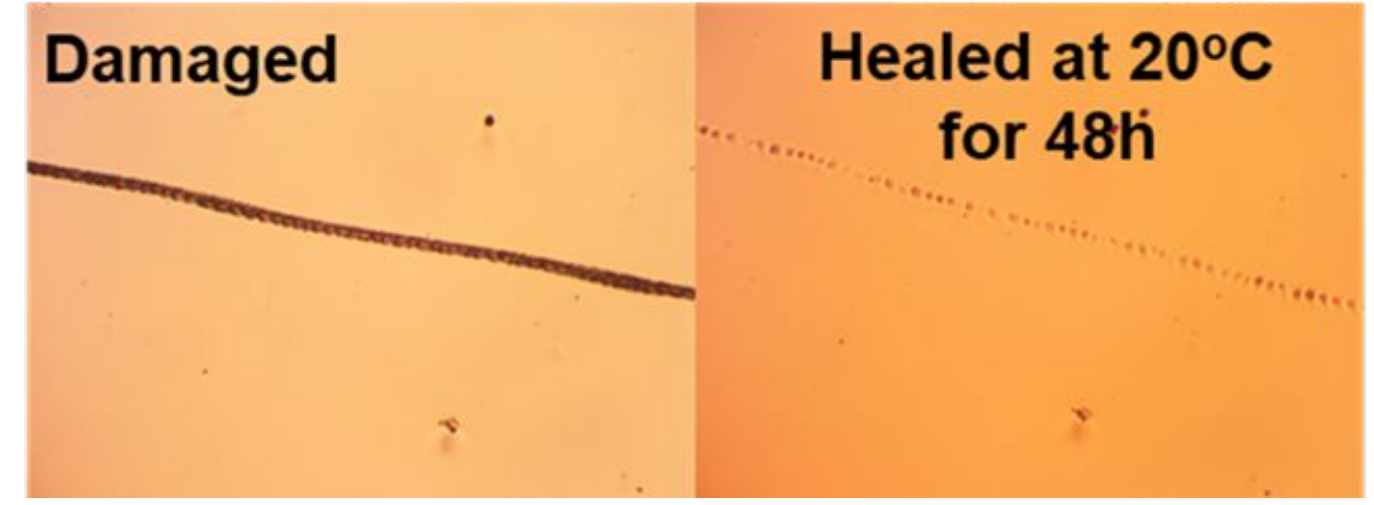

Figure S15. Optical microscopy images of damaged and healed coatings at $20^{\circ} \mathrm{C}$.

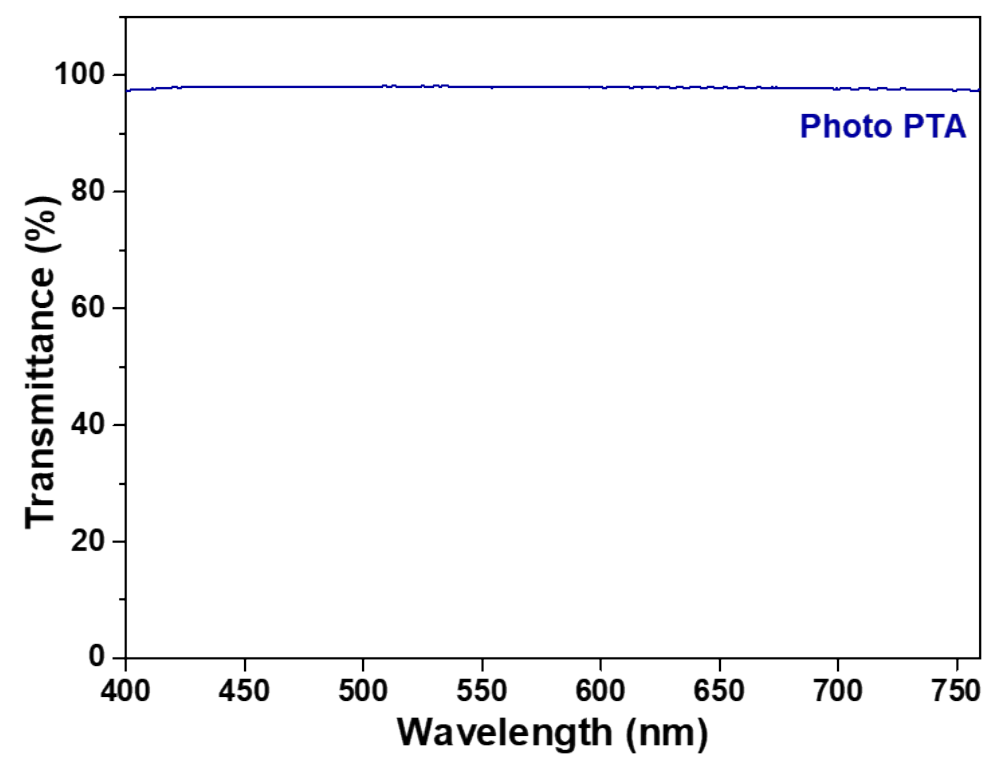

Figure S16. UV-Vis transmission spectrum of photo-curing poly(TA) coating. 